\title{
Ultrafast Beam Switching Using Coupled VCSEL
}

\author{
C.Z.Ning and P. Goorjian \\ Computational Quantum Optoelectronics, NASA Ames Research Center \\ Mail stop T27A-1, Moffett Field, CA 94035-1000, USA \\ Tel.(650)604 3983, Fax (650)604 3957, email:cningenas.nasa.gov
}

\begin{abstract}
We propose a new approach to performing ultrafast beam switching using two coupled Vertical-Cavity Surface-Emitting Lasers (VCSELs). The strategy is demonstrated by numerical simulation, showing a beam switching of 10 degrees at $42 \mathrm{GHz}$.
\end{abstract}




\title{
Ultrafast Beam Switching Using Coupled VCSEL
}

\author{
C.Z.Ning and P. Goorjian \\ Computational Quantum Optoelectronics, NASA Ames Research Center \\ Mail Stop T27A-1, Moffett Field, CA 94035-1000,USA \\ Tel. (650)604 3983, Fax (650)604 3957, email:cningenas.nasa.gov
}

The ability to steer or switch propagation direction of a laser beam in a controllable way is very important for many applications, and especially for optical interconnect networks. Beam scanning and steering in edge emitting lasers have been realized using thermal control [1] and spatial phase controlling technique [2]. For optical interconnect application, all the well-known advantageous attributes of VCSELs make them especially appealing elements for such networks. For this reason, routers integrated together with VCSELs that can be controlled electronically are especially important to reduce the overall volume of interconnect network. By creating a phase shift in part of a VCSEL, it was demonstrated [3] that up to $2 \mathrm{GHz}$ beam switching can be achieved with maximum swing angle of about 3 degree.

In this paper, we propose a different approach to achieve ultrafast beam switching. Our approach is based on two VCSELs put in close proximity of each other. Strong coupling between the two VCSELs through carrier diffusion and through evanescent light produce ultrafast hopping of laser intensity between two VCSELs, resulting an ultrafast far-field switching between two directions. As an example, we demonstrate through extensive numerical simulation that two VCSELs placed about 1 micron apart between the edges can be switched at $42 \mathrm{GHz}$ with maximum swing angles of 10 degree. We also demonstrate that the switching frequency and swing angle can be easily modified by adjusting coupling strength between the VCSELs.

Our simulation begins with the effective Bloch equations (EBEs) as applied to VCSEL simulation in [4]. This model has been used recently to simulate transverse mode dynamics in VCSELs [4]. The model consists of three coupled equations describing diffraction of laser field, carrier diffusion in the transverse plane, and optical gain and refractive index dynamics represented by an effective polarization. In our simulation, the EBE model is solved using finite-difference method in space and time domains directly without assuming a priori any mode structure or number of modes. At a given pumping (DC-bias) level, the simulation is performed over a period of several nanoseconds. The simulation 
result is the space and time resolved complex field amplitude $E(x, y, t)$, from which near- and far-field intensities are obtained. As an example, the simulation is performed for gainguided VCSELs of 5.6 microns in diameter separated 0.8 microns edge-to-edge apart. In all the examples shown in the presentation, both VCSELs are biased slightly above threshold of individual VCSELs. Only one of them is modulated with a sinusoidal signal with an $A C$ amplitude 1 percent of the DC bias.

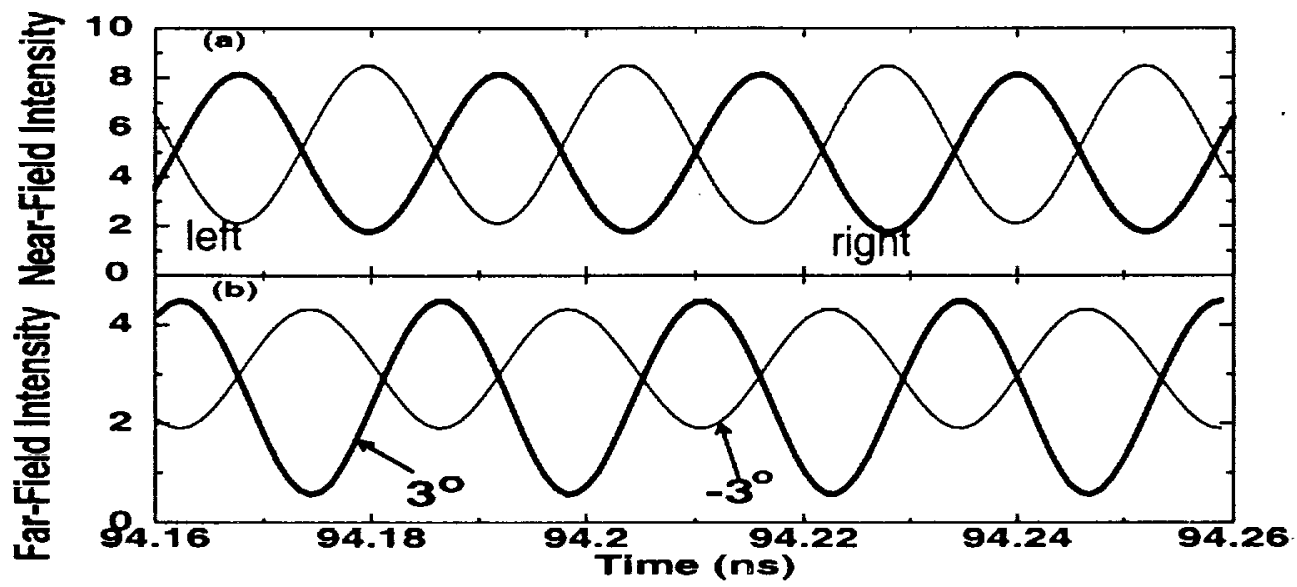

In the figure shown above we plot the time evolution of local laser intensities taken at centers of two VCSELs, as indicated by ' 'left' and 'right'' in the figure. We see clearly that the peak intensities of the two VCSELs are performing anti-correlated oscillation at the modulation frequency of $42 \mathrm{GHz}$. In in the bottom part of the figure (b), the far-field intensity at two angles separated by 6 degrees is plotted, showing again an antiphase oscillation or beam switching between the two angles at 42 GHz. The maximum span of the switching angle is about 10 degrees. Another feature to be noted is that the far field intensity extremes appear when the near field intensities have equal value, showing a maximum constructive interference at an angle to the normal direction of the lasers. As demonstrated clearly in the figure, the beam switching at $42 \mathrm{GHz}$ is related to the hopping of peak intensity between two lasers. This hopping is caused by the coupling between the two VCSELs. To confirm this understanding, we varied the inter-vcsel separation. When the separation decreases, the beam switching frequency increases, and vice versa. At 1 micron separation, the beam switching frequency is about $38 \mathrm{GHz}$. We also studied the influence of DC bias and found that the increase DC current can result an increase in modulation frequency. This is because the increased DC pumping increases the 
spatial carrier overlap between the VCSELs. That, in turn, increases the effective coupling. It is straightforward to conclude that, whenever the coupling strength increases, the beam switching frequency will increase. This obviously gives some design flexibility to engineer the beam switching frequency and angles. For example, if farther separation is desired, an antiguiding type of coupling can be adopted to maintain the strength of coupling and thus the switching frequency. VCSEL arrays based on anti-guiding coupling have been recently demonstrated by several groups. Directional beam switching using anti-guidecoupled VCSELs is currently under study and results will be reported elsewhere.

In Summary, we have proposed a new approach for ultrafast directional beam switching using coupled VCSELs. This approach is demonstrated to achieve over $40 \mathrm{GHz}$ beam switching frequency. Furthermore, we point out that this approach is easy and flexible for design to achieve different switching speed and angle. This approach is likely to be useful for ultrafast optical networks at frequencies much higher than achievable with other approaches. Implementation of this approach for anti-guide coupled VCSELs and other related applications are currently under investigation.

\section{References}

[1]Y. Sun, C.G. Fanning, S.A Biellak, and A.E. Siegman, IEEE Photon. Technol. Lett., $\{\bullet f 7\}, 26(1995)$

[2] J.P. Hohim, D.C. Craft, G.A. Vawter, and D.R. Myers, Appl. Phys. Lett. \{bf 58\}, 2886(1991)

[3]L.Fan, M.C. Wu, and P. Grodzinski, Electronics Letters, 31, $729(1995)$; L.Fan, M.C. Wu, H.C. Lee, and P. Grodzinski, IEEE Photon. Technol. Letters, 9, 505 (1997).

[4] C.Z. Ning and P.M. Goorjian, in Feature Issue on 'Spatial and Polarization Dynamics of Semiconductor Lasers'', eds. G.P.Agrawal, C.Z. Ning, and M. San Miguel, J. Opt. Soc. Am., B16, $2072(1999)$. 

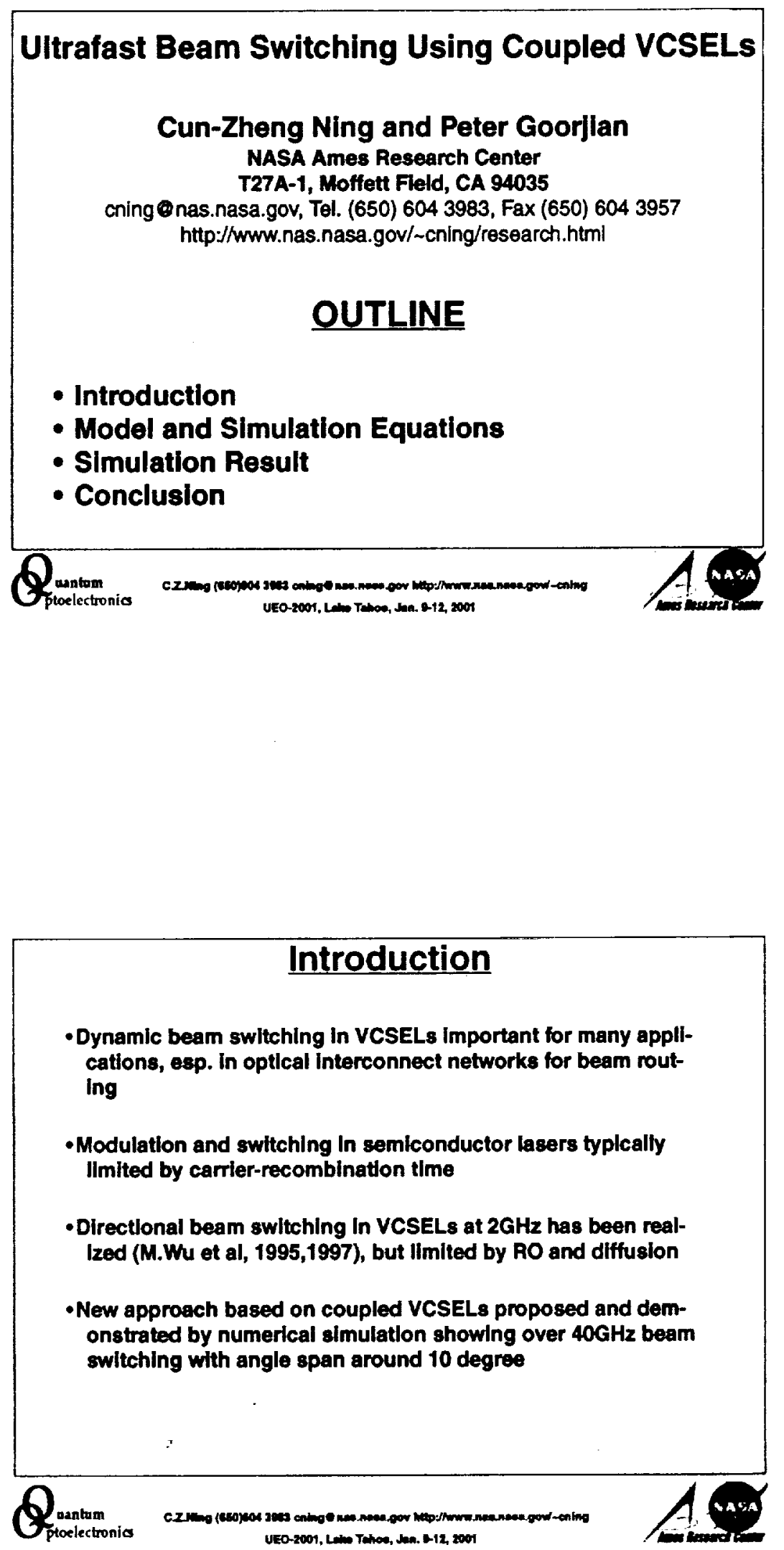

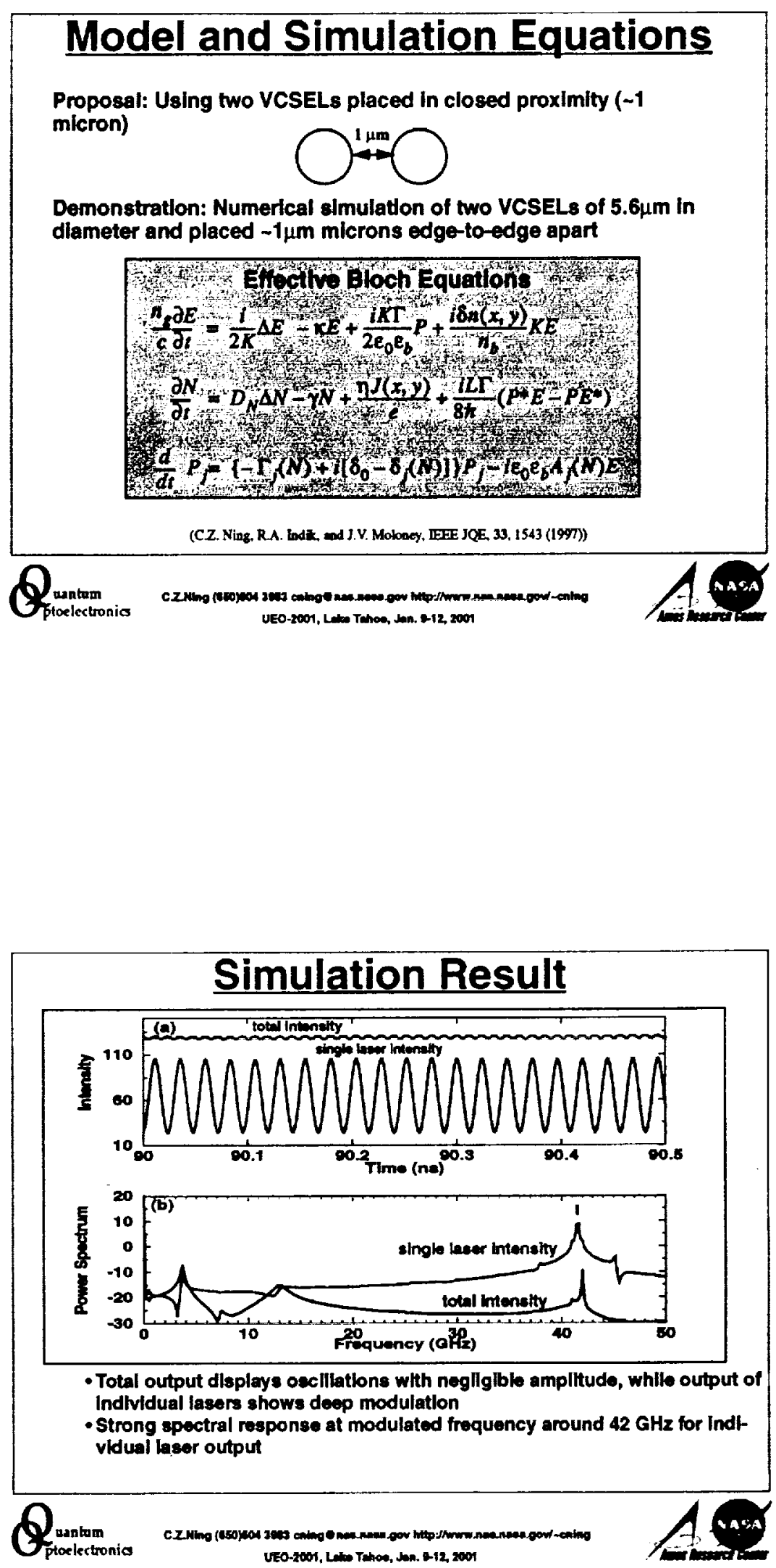


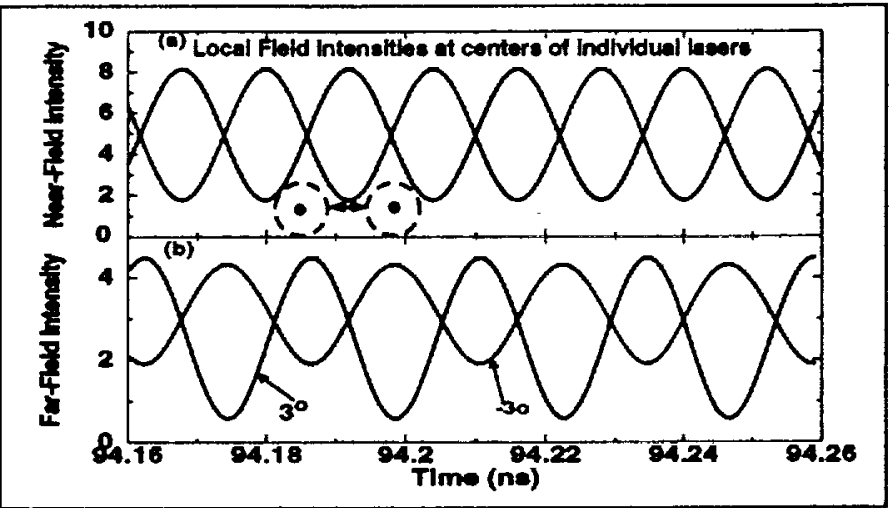

Antiphase oscillation of near field leads to boam switching of far fild at $42 \mathrm{GHz}$

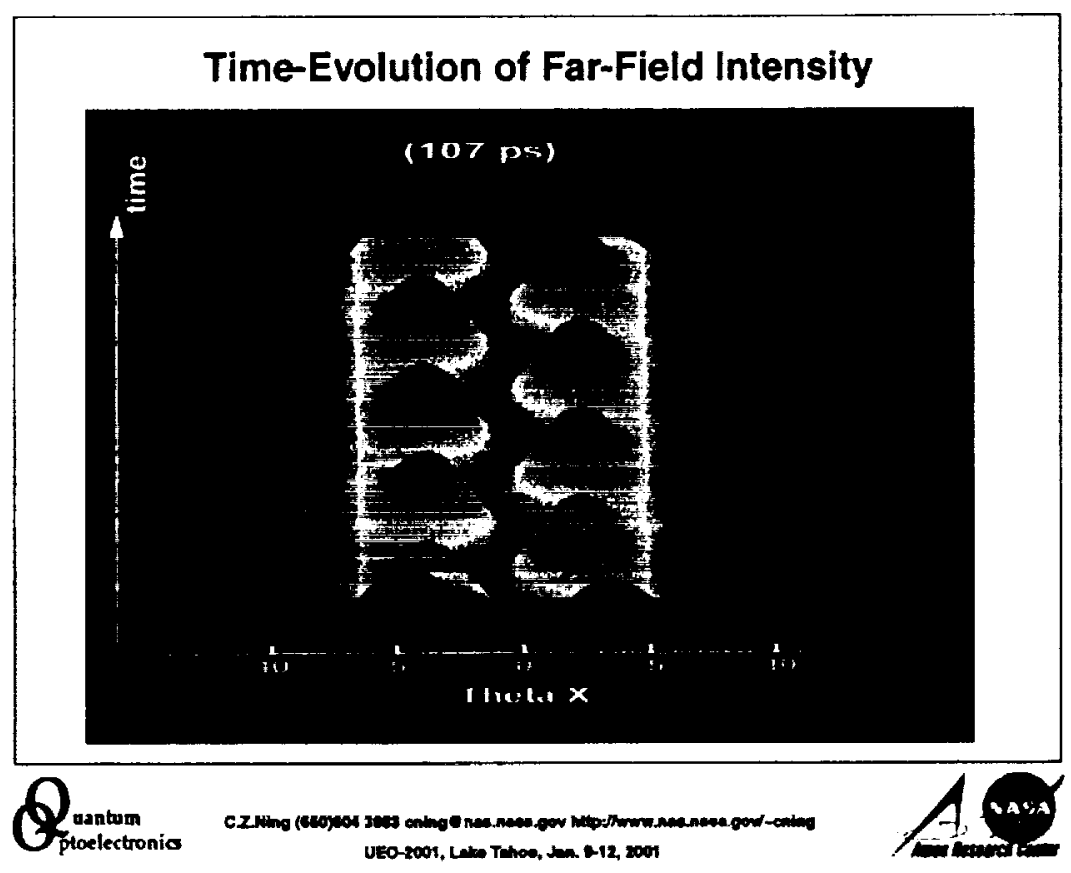



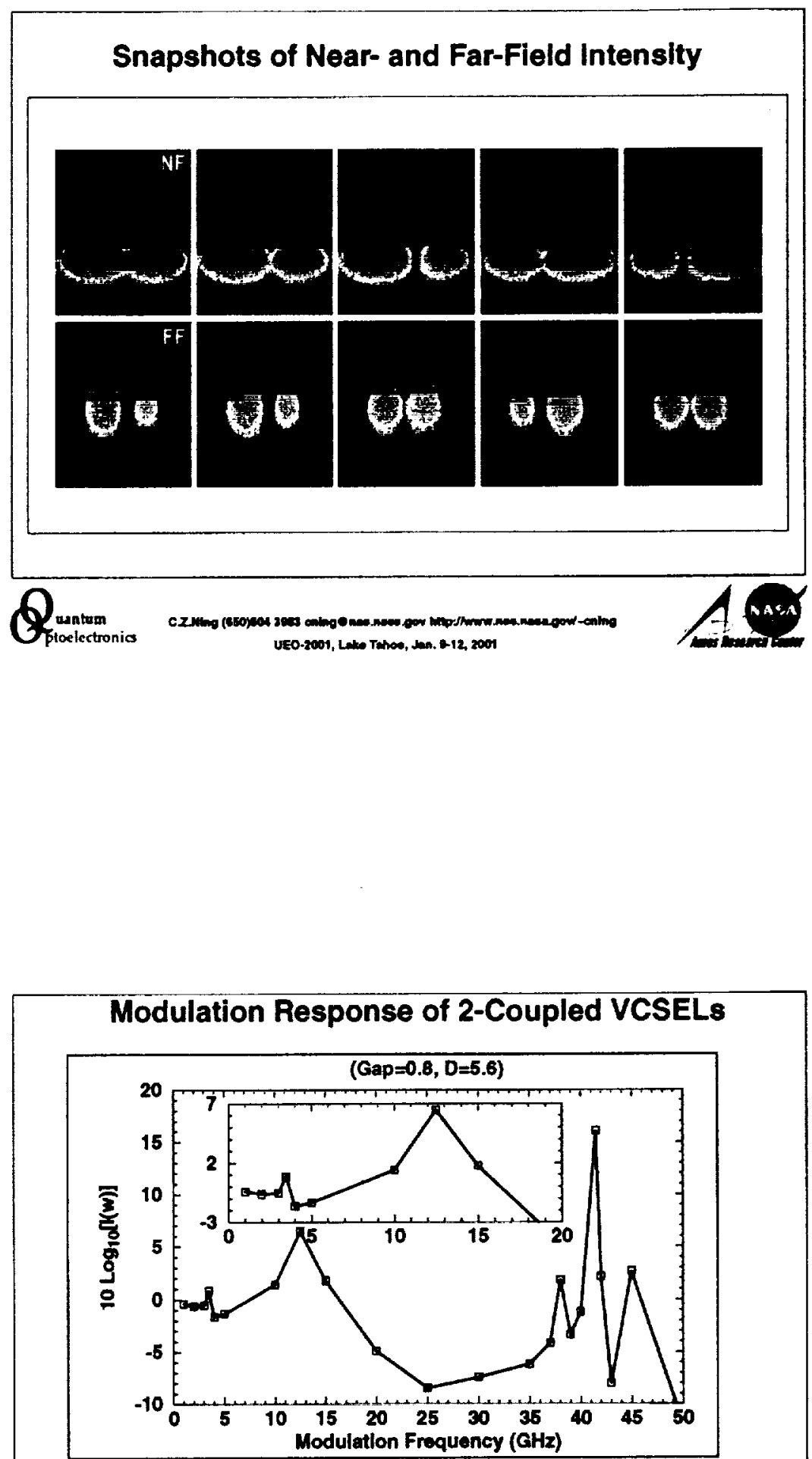

Lower frequency response at 3.5 and $12 \mathrm{GHz}$, related to RO frequencles Strong, narrow bend response at $\mathbf{4 2} \mathbf{~ G H z}$ corresponding to beam switching 


\section{Frequency Change with DC-Blas and Separation}

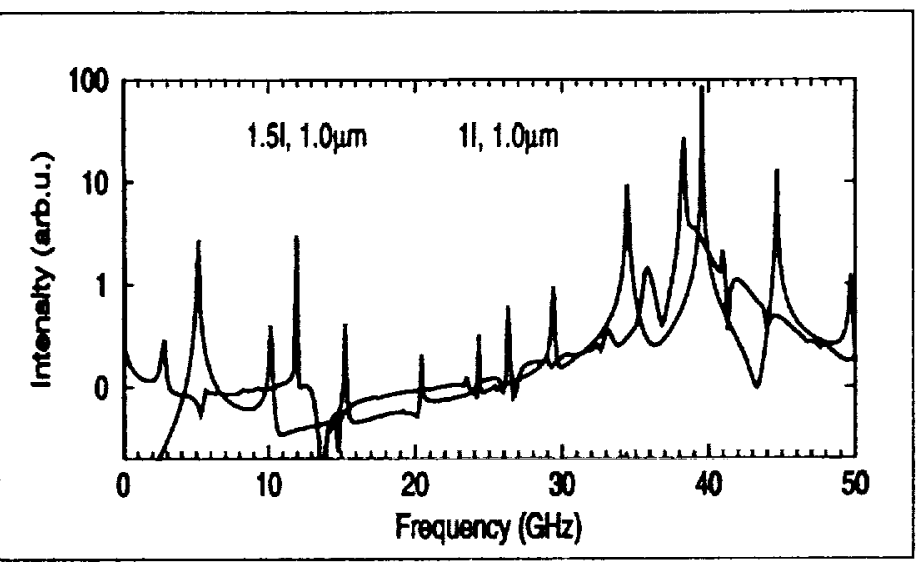

Suntom

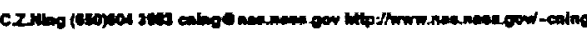

VEO-2001, Lelo Thloo, tan. 2-12, 2001

\section{Conclusion}

- Ultrafast beam switching at frequency > 40GHz with a maximum beam swing angle around 10 degree demonstrated by simulation

- Switching trequency not Ilmited by slow processes such as RO or carrler diffuslon, but controlled by coupling strength between the VCSELs, allowIng englneering of both switching frequency and angle by adjusting coupling strength

Euture and on-aolng work

- Beam swttching using antiguide-coupled VCSELs

- 2D beam routing for 3D networking using following 2D arrangement:

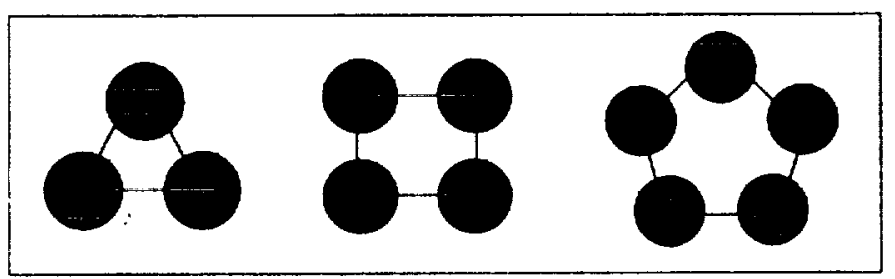

$Q_{\text {matar }}$ 